\title{
Effective Web personalization system using Modified Fuzzy Possibilistic C Means
}

\author{
A. Vaishnavi
}

\begin{abstract}
Due to the rapid growth and development there is an urgent need for the novel technique for the online information system. Web site personalization can be defined as the process of customizing the content and structure of a Web site to the specific and individual needs of each user taking advantage of the user's navigational behavior. A Web personalization system that dynamically suggests interesting URLs for the current user is a major research area of great interest. Clustering plays a very important role in the development of the web personalization. Fuzzy clustering techniques are found to be very efficient in the clustering accuracy. In this paper, a novel technique is proposed for the development of the web personalization system using the Modified Fuzzy Possibilistic C Means (MFPCM). The performance of the proposed Web Personalization system is evaluated based on various parameters. It is observed from the experimental result that the proposed system with MFPCM is effective in providing better and interesting URLs
\end{abstract}

Keywords--- Web Personalization, World Wide Web, MFPCM

\section{INTRODUCTION}

$\mathbf{I}^{\mathrm{T}}$ $\mathrm{T}$ is well known that the World Wide Web may be considered as a huge and global information center. A web site usually contains great amounts of information distributed through hundreds of pages. Without proper guidance, a visitor often wanders aimlessly without visiting important pages, loses interest and leaves the site sooner than expected. This consideration is at the basis of the great interest about web information mining both in the academic and the industrial world. Usually, three types of data have to be managed in a web site: content, structure and log data. Content data consist of whatever is in a web page; structure data refer to the organization of the content; usage data are the usage patterns of web sites. The application of the data mining techniques to these different data sets is at the basis of the three different research directions in the field of web mining: web content mining, web structure mining and web usage mining [1].

The continuous growth in the size and use of the World Wide Web imposes new methods of design and development of online information services. Most Web structures are large and complicated and users often miss the goal of their inquiry, or receive ambiguous results when they try to navigate through them. On the other hand, the e-business sector is

A. Vaishnavi, Gobi Arts and Science, Gobichettipalayam, E-mail: vaishmsc@gmail.com rapidly evolving and the need for Web marketplaces that anticipate the needs of the customers is more evident than ever.

Principal elements of Web personalization include (a) the categorization and preprocessing of Web data, (b) the extraction of correlations between and across different kinds of such data, and (c) the determination of the actions that should be recommended by such a personalization system [10].

The steps of a Web personalization process include: (a) the collection of Web data, (b) the modeling and categorization of these data (preprocessing phase), (c) the analysis of the collected data, and (d) the determination of the actions that should be performed. The ways that are employed in order to analyze the collected data include content-based filtering, collaborative filtering, rule-based filtering, and Web usage mining. The site is personalized through the highlighting of existing hyperlinks, the dynamic insertion of new hyperlinks that seem to be of interest for the current user, or even the creation of new index pages.

One key feature to be addressed in developing a WPS is vagueness and imprecision characterizing Web information space. Besides, it includes a great deal of uncertainty which pervades all stages of the user's interaction: from the definition of the user access model to the recommendation process. Different works have proposed Web Personalization frameworks based on the use of traditional machine learning techniques [8]. However, these techniques may result inefficient and inadequate to handle the uncertainty characterizing the Web interaction processes. These limitations can be overcome through the application of soft computing techniques (i.e. fuzzy logic, neural networks, neuro-fuzzy systems, etc...) which are able to properly deal with the imprecision, uncertainty and partial truths underlying the personalization process [9].

In this paper, we investigate the use of a neuro-fuzzy strategy to develop a Web Personalization framework. In such a framework, a hybrid approach based on the combination of the fuzzy reasoning with a neural network is employed in order to determine user profiles and to provide dynamical predictions about web pages to be suggested to the current user, according to the user profiles previously identified.

\section{RELATED WORKS}

Bouras et al [4] present the dynamic web personalization and document grouping infrastructure for meta-portals and the evaluation of the mechanism on peRSSonal, a system that collects articles from news portals and blogs worldwide. A 
meta-portal is an informational node where articles from different sources are collected and presented in a categorized and personalized manner. The web personalization mechanism is based on dynamic creation and update of user profile according to the user's preferences when browsing and on user grouping details. Assuming that required information, such as article tagging, keywords to categories matching and articles to categories relation is already part of the meta-portal we present a novel mechanism that can build and maintain a user profile which is formed without disturbing the user. Furthermore, we describe the real-time user centered document grouping mechanism that is implemented to support the web personalization system and present the experimental evaluation of the whole system.

Different from most Web recommender systems that are mainly based on clustering and association rule mining, this paper by Wang Xiao-Gang et al., [43] proposes a Web personalization system that uses sequential access pattern mining. In the proposed system an efficient sequential patternmining algorithm is used to identify frequent sequential Web access patterns. The access patterns are then stored in a compact tree structure, called pattern-tree, which is then used for matching and generating Web links for recommendations. In this paper, the proposed system is described, and its performance is evaluated.

There has been a massive growth in systems on the Internet that can personalize the content delivered to individual users. This paper proposed by Ching-Cheng Lee and Wei $\mathrm{Xu}$ [38] develops a category-based Web personalization system in which an unconventional system logger, along with a category generator and a customizer, was designed. This system obtains and records more precise information directly from the users' activity and the objects they access. In addition, the system is designed with its own method to match and deliver individual information in which each user might be interested. Analysis and comparisons with related work are addressed in this research.

\section{Design of Web Personalization System}

In this section, we give an overview of the designed Web personalization system. As illustrated in Figure 1, two major modules can be distinguished in the system: an offline module that extracts knowledge from Web usage data and an online module that recommends interesting Web pages on the basis of the discovered knowledge.

Three main tasks are periodically executed in the offline module:

- Preprocessing of the user access log data to identify user sessions;

- Clustering of the user sessions to generate a number of user profiles.

- Knowledge Extraction to create a set of rules to be used in the recommendation process to determine what URLs have to be primarily suggested to each category of users.

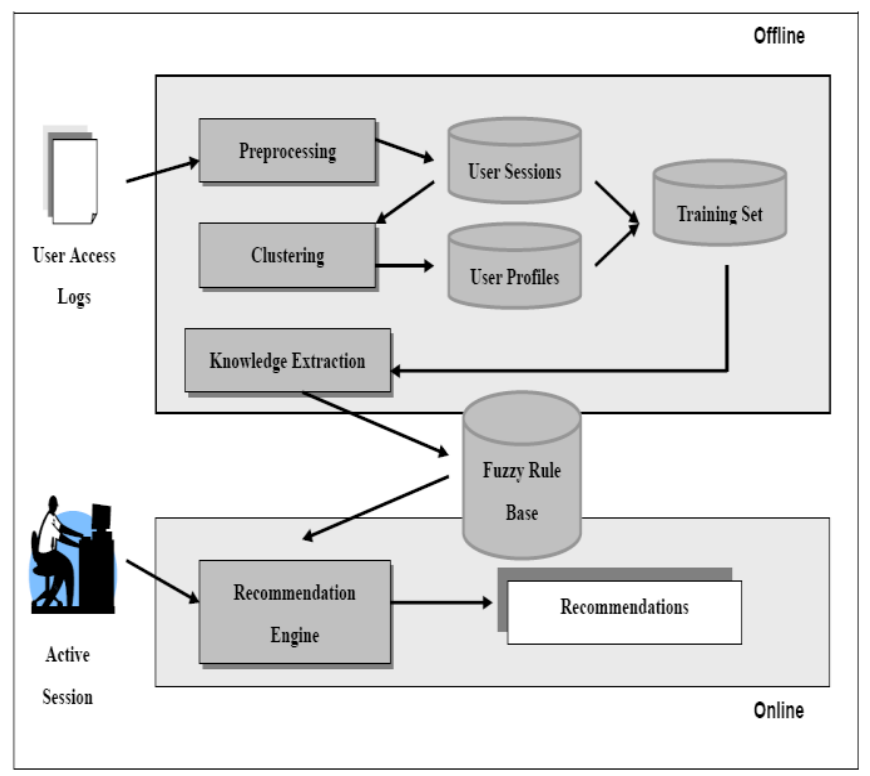

Figure 1: General Scheme of Web Personalization System

In particular, during the preprocessing task, user sessions are extracted from the log files which are stored by the Web server. Each user session is represented by one record which registers the access pattern exhibited by the user in that session. Next, a fuzzy clustering algorithm is executed on these records to group similar sessions into session categories representing user profiles. Finally, starting from the extracted user profiles and the available data about user sessions, a knowledge base expressed in the form of fuzzy rules is extracted via a neuro-fuzzy learning strategy. Such knowledge base is exploited during the recommendation phase (performed by the online module) to dynamically suggest links to Web pages judged interesting for the current user. Specifically, when a user requests a new page, the online module matches his current partial session with the session categories identified by the offline module and derives the degrees of relevance for URLs by means of a fuzzy inference process.

In the following subsections, we describe in more detail all the tasks involved in the Web personalization process.

\subsection{Preprocessing}

When users visit a Web site, the Web server stores the information about their accesses in a log file. Each record of a $\log$ file represents a page request executed from a Web user. In particular, it typically contains the following information (fig. 2): user's IP address, date and time of the access, URL of the requested page, request protocol, a code indicating the status of the request, size of the page (if the request is successful).

$$
\begin{aligned}
& \text { 66.249.65.243 - }- \text { [26/Mar/2006:07:10:44 } \\
& +0200] \\
& \begin{array}{llr}
\text { "GET/gallery2/main.php?g2_view=core.Downlo } \\
\text { adItem\&g2_itemId=5875 } & \text { HTTP/1.1" } & 200
\end{array}
\end{aligned}
$$

Figure 2: An Example of Record in a Log File 
The aim of the preprocessing step is to identify user sessions starting from the information contained in the access $\log$ file. Since sessions encode the navigational behavior of the users, their identification covers an important role for the success of a personalization system. A user session can be defined as a limited set of pages accessed by the same user within a particular visit. According to Nasraoui (2005) and Suryavanshi et al. (2005), we consider a user session as the set of accesses originating from the same IP address within a predefined time period. Such time period is defined as the maximum elapsed time between two consecutive accesses. Supposing that the Web site is composed of $n$ pages, each URL is assigned to a unique number $j=1, . ., n$. Thus, a user session is represented by an $n$-dimensional vector where the $j$ th element expresses the degree of interest of the user for the $j$ th Web page. The degree of interest for a Web page can be defined in different ways. In this work, we consider the degree of interest to a URL as strictly related to the frequency of accesses to the page (number of accesses to that page / total number of accesses during the session) and to the time the user spends on the page. Formally, the $i$-th user session is represented by a vector

$$
\begin{aligned}
& s^{(i)}=\left(s_{1}^{(i)}, s_{2}^{(i)} \ldots ., s_{n}^{(i)}\right) \text { with the property } \\
& s_{j}^{(i)}=\left\{\begin{array}{c}
f_{j}^{(i)} t_{j}^{(i)} \\
0,
\end{array}, \text { if the user visits the } \mathrm{j}\right. \text {-th URL }
\end{aligned}
$$

Otherwise
For $j \in 1, \ldots, n$, where $f_{j}^{(i)}$ and $t_{j}^{\prime \prime}$ indicate, respectively, the access frequency and the time spent by the user on the $j$-th page during the $i$-th session.

Summarizing, after the preprocessing phase, a collection of $N$ sessions $S^{(i)}$ is dentified from the log data.

\subsection{Clustering}

PFCM produces memberships and possibilities simultaneously, along with the usual point prototypes or cluster centers for each cluster. PFCM is a hybridization of possibilistic c-means ( $\mathrm{PCM}$ ) and fuzzy c-means (FCM) that often avoids various problems of PCM, FCM and FPCM. PFCM solves the noise sensitivity defect of FCM, overcomes the coincident clusters problem of PCM. But the noise data have an influence on the estimation of centroids.

The choice of an appropriate objective function is the key to the success of the cluster analysis and to obtain better quality clustering results; so the clustering optimization is based on objective function [16]. To meet a suitable objective function, we started from the following set of requirements:

FPCM algorithm merges the advantages of both fuzzy and possibilistic c-means techniques. Memberships and typicalities are essential for the accurate characteristic of data substructure in clustering technique. Thus, an objective function in the FPCM is based on memberships and typicalities can be given as:

$$
J_{F P C M}(U, T, V)=\sum_{i=1}^{c} \sum_{j=1}^{n}\left(u_{i j}^{m}+t^{n}\right) d^{2}\left(x_{j}, v_{i}\right)
$$

With the following constraints:

$$
\begin{aligned}
& \sum_{i=1}^{c} \mu_{i j}=1, \forall j \in\{1, \ldots, n\} \\
& \sum_{j=1}^{n} t_{i j}=1, \forall i \in\{1, \ldots, c\}
\end{aligned}
$$

The result of the objective function can be achieved through an iterative method where the degrees of membership, typicality and the cluster centers are given by:

$$
\begin{gathered}
u_{i j}=\left[\sum_{k=1}^{c}\left(\frac{d ? x_{j}, v_{i}}{d ? x_{j}, v_{k}}\right)^{2 /(m-1)}\right]^{-1}, 1 \leq i \leq c, 1 \leq j \leq n \\
t_{i j}=\left[\sum_{k=1}^{n}\left(\frac{d ? x_{j}, v_{i}}{d ? x_{j}, v_{k}}\right)^{2 /(n-1)}\right]^{-1}, 1 \leq i \leq c, 1 \leq j \leq n \\
v_{i}=\frac{\sum_{k=1}^{n}\left(u_{i k}^{m}+t_{i k}^{n}\right) x_{k}}{\sum_{k=1}^{n}\left(u_{i k}^{m}+t_{i k}^{n}\right)}, 1 \leq i \leq c
\end{gathered}
$$

FPCM constructs memberships and possibilities concurrently, together with the normal point prototypes or cluster centers for every cluster. The objective function choosing is the very important aspect for the performance of the cluster method and to accomplish better clustering. Therefore the clustering performance is based on objective function to be utilized for clustering. To producing an suitable objective function, the following set of requirements are considered:

- The distance between clusters and the data points allocated to them must be reduced

- The distance between clusters must to be reduced

The desirability between data and clusters is modeled by the objective function. Also Wen-Liang Hung provides a new technique called Modified Suppressed Fuzzy C-Means, which considerably improves the function of FPCM because of a prototype-driven learning of parameter $\alpha$. The learning procedure of $\alpha$ is dependent on an exponential separation strength between clusters and is updated at every iteration. The parameter $\alpha$ is described as:

$$
\alpha=\exp \left(-\min _{i \neq k} \frac{\left\|v_{i}-v_{k}\right\|^{2}}{\beta}\right)
$$

where $\beta$ represents a normalized term so that $\beta$ is taken as a sample variance. That is, $\beta$ is described as:

$$
\begin{aligned}
& \beta=\frac{\sum_{j=1}^{n}\left\|x_{j}-\bar{x}\right\|^{2}}{n} \\
& \text { where } \bar{x}=\frac{\sum_{j=n}^{n} x_{j}}{n}
\end{aligned}
$$

However the statement which must be presented here is the common value used for this parameter by every data at each iteration, which may provided in error. Thus the weight 
parameter is introduced for determining common value for $\alpha$. Or each point of the data set contains a weight in association with each cluster. So the usage of weight allows providing good classification particularly in the case of noise data. So the weight is determined as given below:

$$
w_{j i}=\exp \left(-\frac{\left\|x_{j}-v_{i}\right\|^{2}}{\left(\sum_{j=1}^{n}\left\|x_{j}-\bar{v}\right\|^{2}\right) * c / n}\right)
$$

where $\mathrm{w}_{\mathrm{ji}}$ indicates the weight of the point $\mathrm{j}$ in accordance with the class $i$. This weight is used to alter the fuzzy and typical separation. FPCM technique is iterative in nature, since it is not likely to change any of the objective functions evaluated directly. Otherwise to categorize a data point, cluster centroid has to be nearer to the data point, it is membership; and for determining the centroids, the typicality is used for reducing the undesirable cause of outliers. The objective function contains two expressions:

Fuzzy function and use of fuzziness weighting exponent

Possibililstic function and use of typical weighting exponent

But the two coefficients in the objective function are alone used as exhibitor of membership and typicality. A new relation, slightly unusual, offers a very fast reduction in the function and enhances the membership and the typicality when they inclined near 1 and reduce this degree when they are near 0 . This relation is to afford weighting exponent as exhibitor of distance in the two under objective functions. The objective function of the MFPCM can be described as below:

$$
J_{M F P C M}=\sum_{i=1}^{c} \sum_{j=1}^{n}\left(\mu_{i j}^{m} w_{j i}^{m} d^{2 m}\left(x_{j}, v\right)+t_{i j}^{\eta} w_{j i}^{\eta} d^{2 \eta}\left(x_{j}, v_{i}\right)\right)
$$

$\mathrm{U}=\left\{\mu_{\mathrm{ij}}\right\}$ indicates a fuzzy partition matrix, and is described as:

$$
u_{i j}=\left[\sum_{k=1}^{c}\left(\frac{d ? X_{j}, v_{i}}{d ? X_{j}, v_{k}}\right)^{2 m /(m-1)}\right]^{-1}
$$
as:

$\mathrm{T}=\left\{\mathrm{t}_{\mathrm{ij}}\right\}$ indicates a typical partition matrix, is represented

$$
t_{i j}=\left[\sum_{k=1}^{n}\left(\frac{d ? X_{j}, v_{i}}{d ? X_{j}, v_{k}}\right)^{2 \eta /(\eta-1)}\right]^{-1}
$$

$\mathrm{V}=\left\{\mathrm{v}_{\mathrm{i}}\right\}$ indicates $\mathrm{c}$ centers of the clusters, is represented as:

$$
v_{i}=\frac{\sum_{j=1}^{n}\left(\mu_{i j}^{m} w_{j i}^{m}+t_{i j}^{\eta} w_{j i}^{\eta}\right) * x_{j}}{\sum_{j=1}^{n}\left(\mu_{i j}^{m} w_{j i}^{m}+t_{i j}^{\eta} w_{j i}^{\eta}\right)}
$$

As MFPCM modifies its membership function according to the weight, the segmantation can be performed with better accuracy. When the boundary regions of the cancer nodules are considered, the usage of FPCM will sometimes misjudge the edge of nodule because of its fixed membership function. This problem is overcome by the usage of MFPCM. As the membership function varies according to the weight of a particular region, this helps in reducing the misclassification of borders of the cancer nodule. Some times the cancer nodules will appear in almost same intensity as that of lung region. In this case, the usage of FPCM will not detect the cancer nodule rather it will misclassify the region as lung region. When MFPCM is used, those cancer nodules can be exactly identified because of its capability of changing the membership function.

Summarizing, the clustering phase mines a collection of $C$ session categories from session data, representing profiles of users that have accessed to the Web site under analysis.

\subsection{Knowledge Extraction}

In the knowledge extraction step both session data and session categories identified by fuzzy clustering are employed to extract associations between user sessions and relevance degrees of URLs. Such associations represent the knowledge base to be used in the online recommendation process. The discovery of associations is performed through the learning of a neuro-fuzzy network, i.e. a neural network that encodes in its structure the discovered knowledge in form of fuzzy rules.

In order to learn significant associations, the network has to be trained on a set of input-output samples describing the association between user sessions and preferred URLs. Precisely, the training set is a collection of $N$ input-output vectors:

$$
\mathbf{T}=\left\langle\left(\mathbf{s}^{(i)}, \mathbf{r}^{(i)}\right)\right\rangle_{i=1 \ldots N}
$$

where the input vector $\mathbf{s}(i)$ represents the $i$-th user session identified in the preprocessing phase, and the output vector $r^{(i)}$, expresses the $n$ URLs relevance degrees associated to the $i$-th user session. To compute such relevance degrees, we exploit information embedded in the user profiles extracted through fuzzy clustering. Precisely, for each session $s^{(i)}$, we consider its membership to the $C$ clusters (session categories) expressed by membership values $\left\{m_{i c}\right\}_{c=1 \ldots . . C}$ in the partition matrix $\mathbf{M}$. Then, we identify the two top matching session categories $c_{1}, c_{2} \in\{1, \ldots, C\}$ as those with the highest membership values. The relevance degrees in the output vector $r^{(i)}=\left(r_{1}^{(i)}, \ldots, r_{n}^{(i)}\right)$ are hence calculated as follows:

$$
r_{j}^{(i)}=m_{i, c_{1}} p_{j}^{\left(c_{1}\right)}+m_{i, c_{2}} p_{j}^{\left(c_{2}\right)}
$$

For $\mathrm{j}=1,,,, \mathrm{n}$ and $\mathrm{i}=1, \ldots, \mathrm{N}$

Once the training set has been constructed, the neurofuzzy network can enter the learning phase to extract the knowledge embedded into training set and represent it as a collection of fuzzy rules. Each rule expresses a fuzzy relation between a user session $s=\left(s_{1}, \ldots, s_{n}\right)$ and relevance of URLs in the following form: 
$\mathrm{IF}\left(s_{1}\right.$ is $\left.A_{1 k}\right)$ AND $\ldots$ AND ( $s_{n}$ is $A_{n k}$ ) THEN

(relevance of URL1 is $b_{1 k}$ ) AND ... AND (relevance of $U R L_{n}$ is $b_{n k}$ )

for $k=1, . ., K$ where $K$ is the number of rules, $A_{j k}(j=1, n)$ are fuzzy sets with Gaussian membership functions defined over the input variables $s_{j}$ and $b_{j k}$ are fuzzy singletons expressing the relevance degree of the $j$ th URL.

The fuzzy model is implemented with a three-layer feed forward neural network which reflects the fuzzy rule base in its parameters and topology. The architecture of the network is depicted in fig. 3. In particular, the three layers of the neurofuzzy network compute, respectively:

- membership degree to fuzzy sets;

- fulfillment degree for each fuzzy rule;

- inferred output.

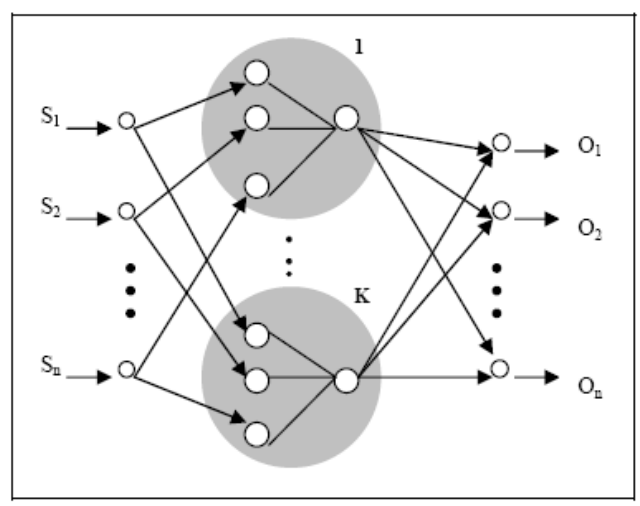

Figure 3: Architecture of the Neuro-Fuzzy Network

Units in the first layer $1 L$ receive the degrees of interest to visited pages in a session $\left(s_{1}, s_{2} \ldots, s_{n}\right)$ and evaluate the Gaussian membership functions representing fuzzy sets. In this layer, units are arranged in $K$ groups, one for each fuzzy rule. The k-th group contains $n$ units corresponding to the fuzzy sets which define the premise part of the k-th rule. In detail, each unit $u_{j k} \in L_{1}$ receives the interest degree for the $\mathrm{j}$ th page $s_{j}, j=1 \ldots . n$ and computes its membership value to fuzzy set $A_{j k}$ as follows

$$
O_{j k}^{(1)}=\exp \left(\frac{\left(s_{j}-x_{j k}\right)^{2}}{\sigma_{j k}^{2}}\right), j=1, \ldots, n k=1, \ldots, K
$$

Where $x_{j k}$ and $\sigma_{j k}$ are the center and the width of the Gaussian function, representing the adjustable parameters of unit $u_{j k} \in L_{1}$.

The second layer $L_{2}$ contains $K$ units that compute the fulfillment degree of each rule. In this layer, no modifiable parameter is associated with the units. The output is derived by computing the rule activation strength, as follows:

$$
O_{j}^{(3)}=\frac{\sum_{k=1}^{K} O_{k}^{(3)} b_{j k}}{\sum_{k=1}^{K} O_{k}^{(3)}}, j=1, \ldots, n
$$

Connections between layer $L_{2}$ and $L_{3}$ are weighted by the fuzzy singletons $b_{j k}$ that represent a set of free parameters for the neuro-fuzzy network. In order to derive the knowledge in the form of fuzzy rule base, a hybrid learning procedure is applied to the neuro-fuzzy network. The neuro-fuzzy learning is articulated in two steps. The first step is based on an unsupervised learning of the neural network, based on a rival penalized mechanism, which provides a clustering of the session data and the definition of an initial fuzzy rule base. In this step, the structure and the parameters of fuzzy rules are identified. Successively, the obtained knowledge base is refined by a supervised learning process. Here, fuzzy rule parameters are tuned via supervised learning to improve the accuracy of the derived knowledge. Major details on the algorithms underlying the neuro-fuzzy learning strategy can be retrieved in Castellano et al. (2005).

\subsection{Recommendation}

The online recommendation module performs the ultimate task of personalization, i.e. suggesting the URLs of the Web site that are judged relevant for the current user. Specifically, when a new user accesses the Web site, the module matches his current partial session against the fuzzy rules obtained offline and derives a vector of relevance degrees by means of a fuzzy inference process. Formally, based on the set of $K$ rules generated through the knowledge extraction procedure described above, the recommendation module provides URL relevance degrees for a new user session $\boldsymbol{s}^{\mathbf{0}}$ by means of the following fuzzy reasoning procedure:

(1) Calculate the matching degree of current session $\boldsymbol{s}^{\mathbf{0}}$ to the $k$-th rule, for $k=1, . ., K$ by means of product operator:

$$
\mu_{k}\left(\mathbf{s}^{0}\right)=\prod_{j=1}^{n} \mu_{j k}\left(s_{j}^{0}\right)
$$

(2) Calculate the relevance degree for the $j$-th URL

$$
r_{j}^{0}=\frac{\sum_{k=1}^{K} r_{j k} \mu_{k}\left(\mathbf{s}^{0}\right)}{\sum_{k=1}^{K} \mu_{k}\left(\mathbf{s}^{0}\right)}, j=1 \ldots n
$$

In order to perform dynamic link suggestion, the recommendation module firstly identifies URLs that have been not visited by the current user, i.e. all pages such that $s^{0}{ }_{j}$. Then, among unexplored pages, only those having a relevance degree $r^{0}{ }_{j}$ greater than a threshold $\alpha$ are recommended to the user by dynamically including a list of links to their URLs in the page currently visited by the user.

\section{CURRENT WORK}

This paper is intended to propose a Web personalization system based on a neuro-fuzzy strategy that is able to dynamically suggest interesting URLs for the current user. The presented system has been designed as composed of two modules: the offline module and the online module. The offline module is devoted to: 
- User session identification by the analysis of Web access log data;

- Categorization of the identified user sessions into user profiles;

- Extraction of a knowledge base through the learning of a neuro-fuzzy network to discover associations between user sessions and relevance degree of URLs.

The online module performs the ultimate recommendation task of the Web personalization system, suggesting the URLs of the Web site that are judged relevant for the current user. In this module, a fuzzy inference process permits to derive a vector of relevance degrees starting from the knowledge base obtained offline. So far, our work has concerned the design and development of the whole recommendation system. Currently, we are starting preliminary simulations needed to identify user session categories and to create the knowledge base for recommendation. To this aim, we are carrying out log data preprocessing to transform access data into an appropriate format, according to the requirements of the clustering algorithm. Two main activities are involved in $\log$ data preprocessing, namely $\log$ data cleaning and session identification. We considered access log files of the Web site of the Boston University Computer Science Department. This $\log$ file, available at http://ita.ee.lbl.gov/html/contrib/BUWeb-Client.html, was collected by the Oceans Research Group at Boston University. It contains a total of 1.143.839 requests, representing a collection of 762 different users. The same log data were used in Xie and Phoha (2001) to derive user profiles by clustering.

\section{EXPERIMENTAL RESULTS}

The proposed approach is evaluated based on the parameters like accuracy, time taken for classification and error rate. The proposed web personalization system uses the modified fuzzy possibilistic $\mathrm{c}$ means clustering algorithm. The previous algorithm uses the fuzzy $\mathrm{c}$ means clustering algorithm.

Thus the performance of the proposed approach is evaluated against the fuzzy c means approach.

\subsection{Classification Accuracy}

The proposed web personalization uses the modified fuzzy possibilistic c means. The accuracy of the proposed web personalization system is compared with the previous web personalization systems which uses the fuzzy c means for clustering. Figure 4 shows the accuracy comparison of the proposed and the existing approaches.

It is observed from the graph that the classification accuracy of the modified fuzzy possibilistic c means is very high when compared to the fuzzy c means.

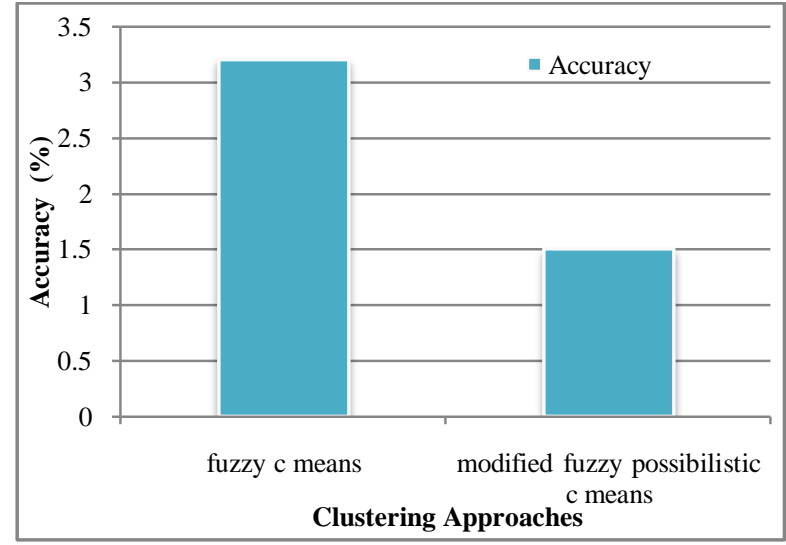

Figure 4: Comparison of Classification Accuracy

\subsection{Time Taken for Classification}

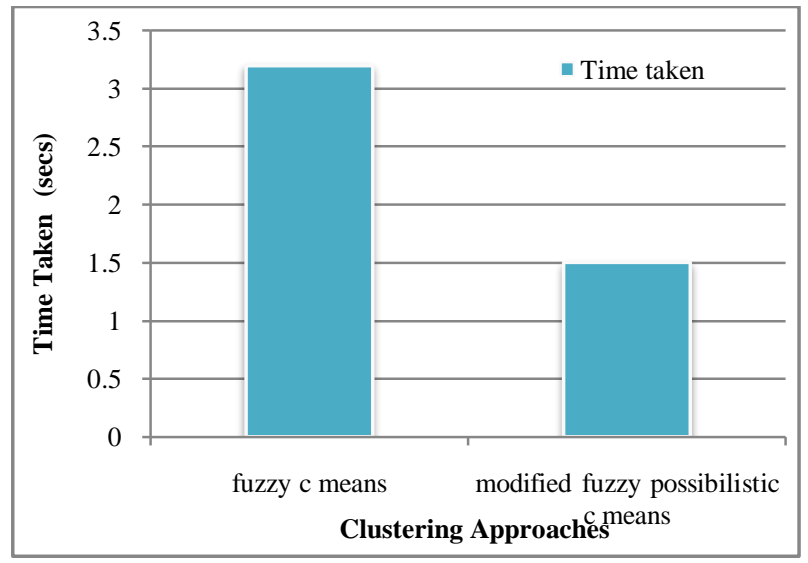

Figure 5: Comparison of Classification Time

Figure 5 shows the graphical representation of the comparison of the time taken for the clustering classification of the fuzzy c means and modified fuzzy possibilistic c means.

It is observed from the graph that the time taken by the modified fuzzy possibilistic c means is very less when compared to the fuzzy c means.

\subsection{Classification Error Rate}

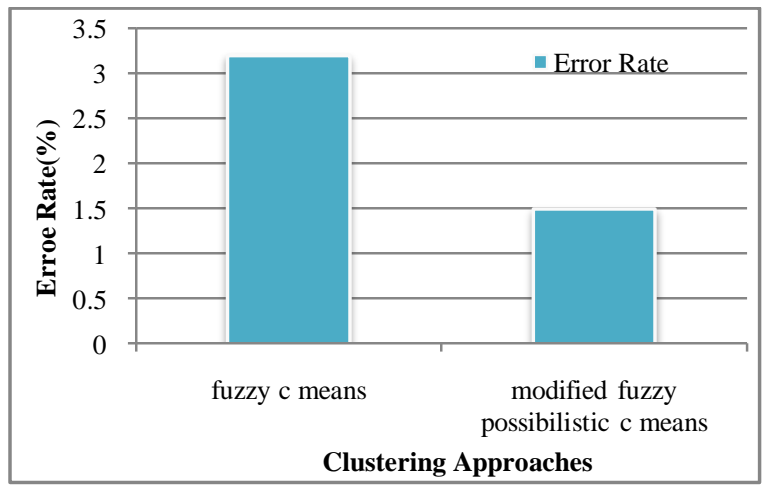

Figure 6: Comparison the Error Rate

Figure 6 shows the graphical representation of the comparison of the error rate for the fuzzy c means and modified fuzzy possibilistic $\mathrm{c}$ means. 
It is observed from the graph that the time taken by the modified fuzzy possibilistic c means is very less when compared to the fuzzy c means.

\section{CONCLUSION}

Thus, because of this MFPCM clustering approach, the proposed web personalization system provides interesting web links. The percentage of the interesting URLs provided to the user is very high. Thus, the proposed web personalization system using the MFPCM is very significant in offering interesting URLs to the users and is making browsing an easier task for the users.

\section{REFERENCES}

[1] F. Zhang and H. Chang. Research and development in web usage mining system-key issues and proposed solutions: a survey. In First IEEE Int. Conf. on Machine Learning and Cybernetics Proceedings, pages 986-990, Nov. 2002.

[2] M. Eirinaki and M. Vazirgiannis. Web mining for web personalization. ACM TOIT., 3(1):2-27, Feb. 2003.

[3] M. Mulvenna, S. Anand, and A. Buchner. Personalization on the net using web mining. CACM, 43(8):123-125, Aug. 2000.

[4] Nasraoui, O. "World Wide Web Personalization", In J. Wang (ed), Encyclopedia of Data Mining and Data Warehousing, Idea Group, 2005.

[5] Mobasher, B., Cooley, R., and Srivastava, J. "Automatic personalization based on web usage mining", Commun. ACM, 43, 8 (August), 142-151, 2000.

[6] Pierrakos, D., Paliouras, G., Papatheodorou, C., and Spyropoulos, C. D. "Web usage mining as a tool for personalization: A survey", User Modeling and User- Adapted Interaction, 13(4), 311-372, 2003

[7] Frias-Martinez, E., Magoulas, G., Chen, S., and Macredie, R. "Modeling human behavior in useradaptive systems: Recent advances using soft computing techniques", Expert Systems with Applications, 29, 320-329, 2005.

[8] Bouras, Christos; Poulopoulos, Vassilis; "Dynamic user context web personalization in meta-portals", IEEE Symposium on Computers and Communications (ISCC), Page(s): 925 - 930, 2010.

[9] Wang Xiao-Gang; Li Yue; "Web mining based on user access patterns for web personalization", ISECS International Colloquium on Computing, Communication, Control, and Management (CCCM), Volume: 1, Page(s): 194 - 197, 2009.

[10] Ching-Cheng Lee and Wei Xu, "Category-based Web personalization system", 25th Annual International Computer Software and Applications Conference (COMPSAC), Page(s): 621 - 625, 2001 